\title{
The Challenge of Predicting Fluid Properties in Carbonate Rocks
}

I. Brevik (StatoilHydro)

\section{SUMMARY}

Predicting fluid phase from geophysical (i.e. normally seismic) data in carbonate reservoir rocks is a challenging task, especially in exploration problems with limited data access and uncertain geological process understanding. This is due to the frequent occurrence of poor seismic amplitude quality in these high velocity and heterogeneous environments, lack of predictive capabilities of rock properties in the complex compaction processes and methodological doubt in how various fluid types and mixes control the seismic properties and in turn the seismic reflectivity caused by carbonate reservoir rocks.

Various examples and a discussion on the concepts of addressing fluid effects in carbonate rocks will be presented. 


\section{Amsterdaml'og}

\section{Introduction}

Predicting fluid phase from geophysical (i.e. normally seismic) data in carbonate reservoir rocks is a challenging task, especially in exploration problems with limited data access and uncertain geological process understanding. This is due to the frequent occurrence of poor seismic amplitude quality in these high velocity and heterogeneous environments, lack of predictive capabilities of rock properties in the complex compaction processes and methodological doubt in how various fluid types and mixes control the seismic properties and in turn the seismic reflectivity caused by carbonate reservoir rocks.

Rock physical methods are needed to understand and predict the effect of various fluid types and mixes on seismic properties in carbonate rocks. Seismic properties, primarily as estimated from seismic inversion, are P-wave impedance and $\mathrm{P}$ to S-wave velocity ratio, or bulk and shear modulus or similar combinations of elastic properties (as estimated from P and S-wave properties and density). P and S-wave velocities are also natural seismic properties, but are not directly obtainable at the resolution needed in prospect evaluation or reservoir characterization problems.

It is well accepted that because of the large stiffnesses associated with carbonate (reservoir) rocks, two different fluid types normally have less impact on seismic properties than in sandstones of similar porosity. The impact difference is due to different compaction processes acting in siliciclastic and carbonate rocks. It is therefore very important to understand and develop schemes enabling modelling of the effect compaction processes have on solid rock and seismic properties in carbonate rocks. The quantification of sensitivity to fluid types and mixes in carbonate rocks is often based on conventional fluid substitution principles applied on wire line well log or core sample data using the Gassmann model. Lately there has been raised concerns and doubt about the validity of Gassmann model in carbonate rocks. On the contrary published analyses of carefully designed experiments demonstrate that this model gives an accurate description of fluid substitution processes in homogenous carbonate rock samples. The concerns have been put forward because some of the fundamental assumptions for the Gassmann model are not met. The assumptions are related to how the model represents the physics of seismic (elastic) wave propagation in porous carbonate rocks saturated with viscous fluids. Recent observations demonstrate effectively that the assumption of a fluid insensitive shear modulus is broken. It is difficult to demonstrate that the other assumptions are broken, although indications and intuitive reasoning lead to doubt about the applicability of Gassmann model.

Alternative rock models addressing the seismic fluid effect have existed for many years. Most of these are based on an explicit representation of a set of non-interacting pores of idealized forms embedded in a homogenous background (like holes in a "swiss cheese"). The pores act as individual sources of (single) scattering of the incoming elastic wave (ultrasonic if core plug experiments), and therefore control the effective velocity. The key challenge is to find a representation of the pores which the elastic waves effectively "see" in porous carbonate rocks. The source of information is traditionally thin slice(s) made from core plugs for which the seismic properties have been measured. The hope is then that this slice represents the whole core plug or even the complete carbonate sequence as represented by accompanying seismic properties (data). The two dimensional thin slices are imaged by using different sources associated with various resolution capabilities. These images are processed to binary representations (pore space and solid, black and white) followed by a fitting process of individual pores to idealized shapes (often ellipses with a specific ratio between the long and short axes, aspect ratio). The final outcome is then an aspect ratio spectrum. If one assumes that the method of individual single scatters is valid, an inversion method using seismic properties as input and aspect ratio spectrum as output might be applied in favourable seismic 


\section{Amsterdam 'og}

amplitude quality cases. Additional constraints in inversion processes can be imposed by quantifying the impact compaction processes have on the pore forms.

Core sample measurements saturated with various fluids and put under relevant stresses/pressure conditions reflecting in-situ conditions represent the traditional method to address and quantify the seismic fluid effect. This method is hampered with a representation uncertainty for practical seismic studies because of the ultra sonic frequencies involved. This uncertainty arises as we cannot assume that low frequent seismic waves "see" the same physical medium as the high frequent waves do. Viscous properties of the fluids will lead to un-relaxed pressures on the pore scale and lead to an effective frequency dependent stiffening of the rock. This velocity dispersion can be large. It is also so that carbonate rocks are rather heterogeneous and we can therefore experience path effects when measuring the travel time of the seismic wave through the rock sample. A recommended alternative is to use longer wavelengths (lower frequencies) in order to measure the effective (total) properties of the rock sample. The low frequency signal source can then not be of the conventional transducer type, but rather obtained by cyclic small strain deformation of the sample where the Young's modulus and Poisson's ratio are monitored.

Well log data analysis are often preferred to address the seismic fluid effect, but due to problems of finding the same background (solid) rock saturated with two different fluids (or mixes) in a carbonate reservoir sequence, it is almost impossible to map with precision the true seismic fluid effect. We are left with numerical fluid substitution schemes, in addition to including possible chemical solid-fluid effects which act on the solid part of the carbonate rocks.

Recommendations for R\&D and practical seismic fluid effect evaluation schemes will be discussed. 